\title{
Fiber composite materials via coaxial, dual or blend electrospinning
}

\begin{abstract}
Electrospinning (ES) is a suitable and cost effective method to mimic the chemical composition, morphology, and functional surface of natural tissues, for example of the nervous, dermal, vascular, and musculoskeletal systems. This technique is a versatile tool to obtain tailored fibrous scaffolds from various polymer materials. By varying the diameter, porosity, orientation, layering, surface structuring, mechanical properties and biodegradability of the fibers the properties can be adapted for specific applications ranging from implantable medical devices to wound repair and protective clothing. Especially the combination of different polymer types offers a high potential. In this study electrospun two-component nonwoven structures of thermoplastic copolyester elastomer (TPC-ET) and bioresorbable polylactide (PLLA) were fabricated, using different ES setups. A comparative evaluation in terms of porosity, thermal and mechanical properties as well as required fabrication effort, was performed. Nonwovens made from polymer blends and coaxial spun core-sheath fibers showed similar tensile strength, which was higher than dual electrospun fabrics. Porosity was found to be in the range of $80-90 \%$. By modifying the polymer solution and process parameters multicomponent nonwoven structures with tailored properties and drug release profiles can be manufactured.
\end{abstract}

Keywords: core-sheath, nanofiber, nonwoven, scaffold.

https://doi.org/10.1515/cdbme-2021-2173

\section{Introduction}

There is an increasing interest in ultra-fine or highly porous polymeric electrospun nonwovens carrying bioactive

\footnotetext{
*Corresponding author: Sabine Illner: Institute for Biomedical Engineering, Rostock University Medical Center, 18119 RostockWarnemünde, Germany, e-mail: sabine.illner@uni-rostock.de Michelle Sühr, Nicklas Fiedler, Daniela Arbeiter, Andreas Götz, Klaus-Peter Schmitz and Niels Grabow: Institute for Biomedical Engineering, Rostock University Medical Center, 18119 Rostock-Warnemünde, Germany
}

substances for usage as drug delivery systems as well as basic structures fabricated on 3D-moldings for temporary or permanent implants.[1,2]

Nonwoven structures are already used to cover implants. Further applications include barrier membranes to prevent postoperative adhesion, filter membranes to separate bacteria and blood cells, wound dressings, and developments or improvements of biomedical devices.[2] New material properties are created by combining polymers to better mimic native tissues. In this regard multicomponent scaffolds may represent the most advantageous combination of material properties for various applications.

In this context, it is important to mention that ES is a complex process, with an extensive parameter set that cannot be defined in general terms. An individual set of parameters must be defined for each polymer (see Table 1).

Table 1: Parameters of impact in electrospinning.

\begin{tabular}{|c|c|c|}
\hline $\begin{array}{l}\text { Polymer solution } \\
\text { properties }\end{array}$ & $\begin{array}{l}\text { Process } \\
\text { parameters }\end{array}$ & $\begin{array}{l}\text { Environmental } \\
\text { conditions }\end{array}$ \\
\hline Molecular weight & High voltage & Temperature \\
\hline Viscosity & Flow rate & Humidity \\
\hline Surface tension & $\begin{array}{l}\text { Geometry of } \\
\text { emitter/collector }\end{array}$ & $\begin{array}{l}\text { Atmospheric } \\
\text { composition }\end{array}$ \\
\hline Conductivity & $\begin{array}{l}\text { Emitter-collector } \\
\text { distance }\end{array}$ & Air/gas flow \\
\hline
\end{tabular}

The polymer concentration and molecular weight are of greatest influence on the ES process and fiber morphology. Furthermore, a balanced adjustment of voltage and flow rate is necessary for a stable process. To produce a continuous jet, the applied voltage must be high enough to overcome the surface tension of the solution at the end of the Taylor cone, whereby the jet is most stable just above the critical voltage. Since all parameters influence each other, it is advantageous to keep some parameters, such as temperature and humidity, constant to reach homogeneous fiber formation.

Coaxial ES was introduced as a special method in 2003 and is based on the simultaneous spinning of two polymeric solutions through a so-called coaxial spinneret.[3] Blend ES of different polymer types in one solution is often limited by 
the solubility of different polymers.[4] Dual ES is suggested in particular to produce biomimetic nonwovens.[5]

Being an integral part of ES quality assurance, morphological observations using scanning electron microscopy provide information about the relationship between fiber structure and process parameters. Also, the interest in a fast, error-free, intelligent and automatable evaluation of such nanofiber structures is getting into focus.[6]

The objective of this work was to characterize and compare electrospun two-component nonwoven structures using different ES setups and polymers. Due to its biocompatibility and biodegradability, poly(L-lactide) PLLA is frequently used in medical technology, e.g. it is applied for drug release in stent coatings. PLLA is characterized by high Young's modulus and tensile strength, scratch resistance, transparency, and chemical resistance. Thermoplastic copolyester elastomer TPC-ET has a wide range of applications due to its combination of high flexibility of elastomers and the strength of thermoplastics. TPC-ET is characterized by mechanical durability and flexibility, creep resistance, as well as thermal and chemical resistance. Therefore, it is used among others, in automotive components, fluid power, electronics, consumer products, appliance and power tools, and sports equipment.

\section{Materials and methods}

\subsection{Fiber material fabrication}

Non-biodegradable TPC-ET and bioresorbable PLLA were chosen to produce fiber blend and composite materials. The polymers used were selected for their ease of spinning, glare resistance and different shore hardness.

A polymer solution of $4.5 \mathrm{wt} \%$ PLLA (RESOMER L210, Evonik, Germany) and $6.5 \mathrm{wt} \%$ TPC-ET (Hytrel 30D, DuPont, France) was obtained by dissolving in chloroform and 2,2,2-trifluoroethanol $\left(1: 4, \mathrm{v} / \mathrm{v}\right.$ ) at $37^{\circ} \mathrm{C}$ (lab shaker at $100 \mathrm{rpm}$ ) for 1 to 2 days. Two different core-sheath fibers were prepared with the same polymer solutions, where PLLA was either fed in the core or in the sheath, and being complemented by TPC-ET, respectively. Polymer blend was prepared of PLLA/TPC-ET in a ratio of $2.25 \% / 3.25 \%$.

The 4SPIN C4S LAB2 device (Contipro, Dolní Dobrouč, Czech Republic), which includes a home-built rotating continual collector, was used for the ES process. Different polyester composites were processed by coaxial, dual and blend ES setups using different parameter sets and multi-jet capillary emitter systems. The emitter-collector distance was kept constant at $25 \mathrm{~cm}$ whereas the voltage and the feed rate were adapted to different requirements of the emitter. The spinning process was performed using ambient temperature of $22 \pm 2^{\circ} \mathrm{C}$ and humidity of $25 \pm 5 \%$.

\subsection{Material characterization}

The material characteristics were investigated in terms of morphology, thermal and mechanical properties, as well as porosity, using the scanning electron microscope Quanta FEG 250 REM (FEI Company, Germany). Gravimetry was performed using a precision balance (Kern, Germany) and dial gauge 2046SL (Mitutoyo, Kawasaki, Japan).

\subsubsection{Porosity calculation (air content)}

Round samples with a diameter of $9 \mathrm{~mm}$ were punched out of the produced nonwovens $(n=3)$. For comparison the density of solid material was used as reference, TPC-ET $\left(1.07 \mathrm{mg} / \mathrm{mm}^{3}\right)$ and PLLA $\left(1.27 \mathrm{mg} / \mathrm{mm}^{3}\right)$. Taking into account the quantity ratios and assuming that the solvents are completely removed as well as the density of the polymer does not change during processing, the porosity of nonwovens was calculated according to the following equation:

$$
P=1-\frac{\frac{m}{\left(\frac{D}{2}\right)^{2} * \pi * d}}{f_{1} * \rho_{1}+f_{2} * \rho_{2}}
$$

with $\mathrm{P}$ - porosity (air content), $\mathrm{m}$-mass of sample, D diameter of sample, $d$ - thickness of sample, $f_{1}$ - fraction of polymer $1, \rho_{1}-$ density of polymer $1, f_{2}-$ fraction of polymer $2, \rho_{2}$-density of polymer 2 .

\subsubsection{Thermal and mechanical testing}

The tensile tests were performed using a universal testing machine Zwick BZ2.5/TN1S (Zwick/Roell, Ulm, Germany). The produced nonwovens were investigated with regard to their application as implant material in $0.9 \%$ saline solution at $37^{\circ} \mathrm{C}$.

Prior to testing, samples were cut out of the nonwovens and stored in $0.9 \%$ saline for at least 24 hours to wet equilibrium state for simulation as implant material in the body. Since changes in the size of the sample can occur during the soaking of the materials, the shoulder bars $(n=3)$ 
were not punched out in standardized sample design (1BB, DIN EN ISO 527-2:2012) until shortly before the tensile test was performed. A subset of the patches was perforated in a region of $\pm 10 \mathrm{~mm}$ around their center. The test patches were mounted at a $10 \mathrm{~N}$ load cell and extended with $25 \mathrm{~mm} \cdot \mathrm{min}^{-1}$.

Thermal measurements according DIN EN ISO 11357 were performed at $-50^{\circ} \mathrm{C}$ to $200^{\circ} \mathrm{C}$ using a DSC1 $\mathrm{Star}^{\mathrm{e}}$ system (Mettler Toledo, Germany). For this purpose, small samples $(n=3)$ were cut out of the nonwovens, the mass was determined with the aid of the fine scale, and then placed in an aluminum crucible with a lid and compressed. The crucible was placed in the oven and then examined at a temperature interval of $-50^{\circ} \mathrm{C}$ to $200^{\circ} \mathrm{C}$.

\section{Results and discussion}

\subsubsection{Morphology and porosity}

High process stability and unaligned, slightly bonded beadfree fibers (without thickening or bead structure) were achieved for all composites investigated (see Figure 1).

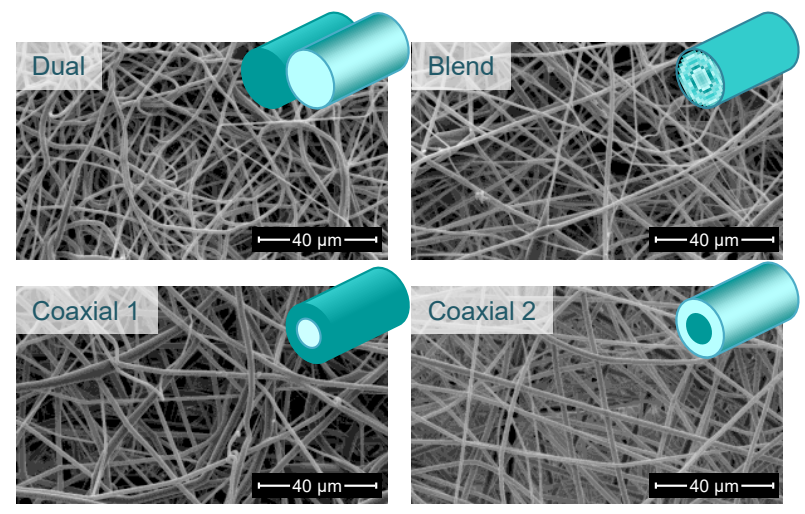

Figure 1: SEM images of electrospun two-component nonwovens with schematically shown fiber type, each right top (1000x magnification).

Homogeneous and isotropic nonwoven materials were obtained. Fiber diameters of approximately one micrometer were realized for TPC-ET and PLLA nonwovens; thus preventing fiber differentiation. The same is observed for blend and dual electrospun nonwovens. In contrast, thicker fibers are obtained using coaxial ES, which indicates a successful formation of core-sheath fibers. Overall, it was found that fiber diameters were slightly increased on the top side of the nonwoven as opposed to the bottom side (data not shown). This indicates that the process is not yet sufficiently initiated and that air may have been entrained in the system at the beginning. Thinner fibers usually require higher elastic moduli, which makes comparison of electrospinning techniques more difficult. This observation can serve quality assurance in the future. Therefore nonwovens demonstrating fiber diameter deviations of more than $20 \%$ should be excluded.

Porosity was found to be in the range of $80-90 \%$ (see Figure 2), which is consistent with literature data for nonwoven structures.[7] Thus, the determined porosity of the nonwovens is comparable to that of sponges.[8]

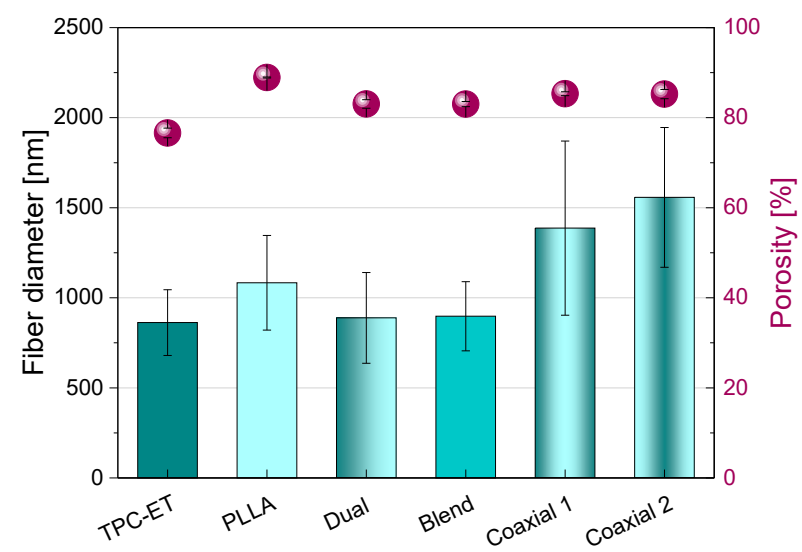

Figure 2: Fiber diameter (top side, $n \geq 10$ ) and porosity of twocomponent nonwovens.

The lowest porosity of the nonwoven structures produced is shown by the TPC-ET nonwoven with a value of $77 \%$. The PLLA nonwoven, on the other hand, has the highest air content with $89 \%$. The values of two-component nonwoven structures are in between and range from $80-85 \%$.

\subsubsection{Tensile strength and thermal analysis}
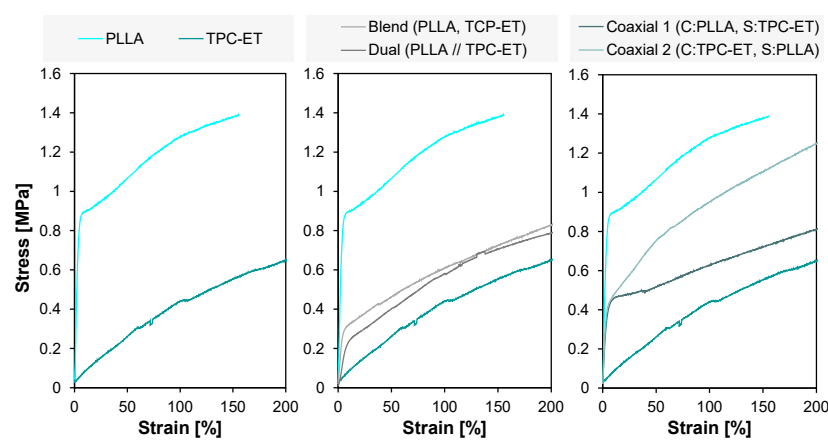

Figure 3: Stress-strain curves of electrospun single or twocomponent nonwoven samples $(n=3)$, measured in $0.9 \%$ saline at $37^{\circ} \mathrm{C}$ (representative section up to $200 \%$ elongation). 
Nonwovens fabricated from polymer blends and coaxial spun core-sheath fibers exhibited similar tensile strength in $0.9 \%$ saline at $37^{\circ} \mathrm{C}$, which was higher than for dual electrospun fabrics. Figure 3 shows that similar stress-strain curves are realized in blend and dual ES. In coaxial spinning, the sheath has a decisive influence on tensile strength and elongation.

In general, nonwoven materials are not at all comparable with foils due to their high porosity. For illustration purposes, Table 2 compares the most important thermal and mechanical properties of the raw and nonwoven materials.

Only the melting and glass transition temperature of the two-component nonwoven structures correspond to those of pure PLLA. In addition, an earlier increase of the melting range is recognizable.

Table 2: Material data - mechanical $\left(n=5,37^{\circ} \mathrm{C}\right.$, wet $)$ and thermal $(n=3)$ properties. (Abbr.: $E$ - tensile strength (Young's modulus), $\varepsilon$ - elongation at break, $\mathrm{T}_{\mathrm{m}}$ - melt temperature, $\mathrm{T}_{\mathrm{g}}$ - glass transition temperature, 1.HC - data of first heat curve, * raw material, data of supplier).

\begin{tabular}{lllll}
\hline Polymer & $\mathbf{E}[\mathbf{M P a}]$ & $\boldsymbol{\varepsilon}[\%]$ & $\mathbf{T}_{\mathbf{m}, 1 . \mathrm{Hc}}\left[{ }^{\circ} \mathbf{C}\right]$ & $\mathbf{T}_{\mathbf{g}, \text { 1.Hc }}\left[{ }^{\circ} \mathbf{C}\right]$ \\
\hline PLLA $^{*}$ & $28-50$ & $6[9]$ & $180-185$ & $60-65$ \\
TPC-ET $^{*}$ & 24 & $>300$ & 177 & - \\
\hline PLLA & $15-35$ & $>150$ & 178 & 63 \\
TPC-ET & $0.3-0.9$ & $>700$ & 163 & $(54)$ \\
\hline Blend & $4.8-9.1$ & $>350$ & 177 & 62 \\
Dual & $1.4-3.1$ & $>800$ & 178 & 64 \\
Coaxial 1 & $10-12$ & $>500$ & 177 & 63 \\
Coaxial 2 & $10-17$ & $>300$ & 177 & 63 \\
\hline
\end{tabular}

\section{Conclusion}

In a systematic investigation the combination of nonbiodegradable and bioresorbable polyester with entirely different mechanical properties and application potentials was investigated. By varying the polymer solution composition and the ES setup, two-component nonwoven structures with tailored properties in terms of flexibility and interconnection of the fibers can be obtained through a more or less complex manufacturing process. Each ES setup shows its advantages and disadvantages in terms of process time and flexibility with regard to mechanical target parameters.

In this context, the time aspect for using a coaxial nozzle in contrast to multiple nozzle systems (with needles or needleless) for blend or dual electrospinning should be considered, as well. From a process point of view the application of coaxial spinning appears to be particularly advisable for specific (intelligently controllable) active pharmaceutical ingredient incorporation and multimodal release.

\section{Author Statement}

Research funding: Partial financial support by the Federal Ministry of Education and Research (BMBF) within RESPONSE "Partnership for Innovation in Implant Technology" is gratefully acknowledged.

The Authors sincerely thank B. Hummel, K. Hahn, J. Ortelt, P. Reichelt and M. Strotmeier for their skillful work.

Conflict of interest: Authors state no conflict of interest.

\section{References}

[1] Bhardwaj N, Kundu SC. Electrospinning: a fascinating fiber fabrication technique. Biotechnol Adv. 2010;28:325-347.

[2] Xue J, Wu T, Dai Y, et al. Electrospinning and Electrospun Nanofibers: Methods, Materials, and Applications. Chem Rev. 2019;119:5298-5415.

[3] Sun Z, Zussman E, Yarin AL, et al. Compound Core-Shell Polymer Nanofibers by Co-Electrospinning. Adv. Mater. 2003;15:1929-1932.

[4] Basu P, Repanas A, Chatterjee A, et al. PEO-CMC blend nanofibers fabrication by electrospinning for soft tissue engineering applications. Materials Letters. 2017;195:10-13.

[5] Illner S, Arbeiter D, Teske M, et al. Tissue biomimicry using cross-linked electrospun nonwoven fibre composites. Current Directions in Biomedical Engineering. 2019;5:119-122.

[6] Götz A, Senz V, Schmidt W, et al. General image fiber tool: A concept for automated evaluation of fiber diameters in SEM images. Measurement. 2021;177:109265.

[7] Rasouli R, Barhoum A, Bechelany M, et al. Nanofibers for Biomedical and Healthcare Applications. Macromol Biosci. 2019;19:e1800256.

[8] Zhang $\mathrm{H}$, Jin $\mathrm{Y}$, Chi C, et al. Sponge particulates for biomedical applications: Biofunctionalization, multi-drug shielding, and theranostic applications. Biomaterials. 2021;273:120824.

[9] El-Hadi AM. Increase the elongation at break of poly (lactic acid) composites for use in food packaging films. Sci Rep. 2017;7:46767. 